\title{
Defensive Alliances in Graphs of Bounded Treewidth*
}

\author{
Bernhard Bliem and Stefan Woltran \\ Institute of Information Systems 184/2 \\ TU Wien \\ Favoritenstrasse 9-11, 1040 Vienna, Austria \\ [bliem, woltran] @dbai.tuwien.ac . at
}

\begin{abstract}
A set $S$ of vertices of a graph is a defensive alliance if, for each element of $S$, the majority of its neighbors is in $S$. The problem of finding a defensive alliance of minimum size in a given graph is NP-hard and there are polynomial-time algorithms if certain parameters are bounded by a fixed constant. In particular, fixed-parameter tractability results have been obtained for some structural parameters such as the vertex cover number. However, for the parameter treewidth, the question of whether the problem is FPT has remained open. This is unfortunate because treewidth is perhaps the most prominent graph parameter and has proven successful for many problems. In this work, we give a negative answer by showing that the problem is W[1]-hard when parameterized by treewidth, which rules out FPT algorithms under common assumptions. This is surprising since the problem is known to be FPT when parameterized by solution size and "subset problems" that satisfy this property usually tend to be FPT for bounded treewidth as well. We prove $\mathrm{W}[1]$-hardness by using techniques from a recent hardness result for the problem of finding so-called secure sets in a graph.
\end{abstract}

1998 ACM Subject Classification F.2.2 Nonnumerical Algorithms and Problems

Keywords and phrases defensive alliance, alliances in graphs, treewidth, complexity analysis, parameterized complexity

\section{Introduction}

The objective of many problems that can be modeled as graphs is finding a group of vertices that together satisfy some property. In this respect, one of the concepts that has been quite extensively studied is the notion of a defensive alliance [28, 27], which is a set of vertices such that for each element $v$ at least half of its neighbors are also in the alliance. The name "defensive alliance" stems from the intuition that the neighbors of an element $v$ that are also in the alliance can help out in case $v$ is attacked by its other neighbors.

Notions like this can be applied to finding groups of nations, companies or individuals that depend on each other, but also to more abstract situations like finding groups of websites that form communities [18]. Another possible application for defensive alliances are computer networks, where a defensive alliance represents computers that can provide a certain desired resource; any computer in an alliance can then, with the help of its neighbors that are also in the alliance, allow access to this resource from all of its neighbors simultaneously [20].

Several variants of defensive alliances have also been studied. The papers that originally proposed defensive alliances also propose related notions like offensive and powerful alliances. An offensive alliance is a set $S$ of vertices such that every neighbor of an element of $S$ has at

* This work was supported by the Austrian Science Fund (FWF) projects P25607 and Y698.

(c) (i) () Bernhard Bliem and Stefan Woltran;

licensed under Creative Commons License CC-BY

Leibniz International Proceedings in Informatics

LIPICS Schloss Dagstuhl - Leibniz-Zentrum für Informatik, Dagstuhl Publishing, Germany 
least half of its neighbors in $S$, and a powerful alliance is both a defensive and an offensive alliance. Any of these alliances is called global if it is at the same time a dominating set. Another variant is to consider alliances $S$ where, for each vertex $v \in S$, the difference between the number of neighbors of $v$ in $S$ and the number of other neighbors of $v$ is at most a given integer [31]. For comprehensive overviews of different kinds of alliances in graphs, we refer to the surveys [34, 17].

The Defensive Alliance problem can be specified as follows: Given a graph $G$ and an integer $k$, is there a defensive alliance $S$ in $G$ such that $1 \leqslant|S| \leqslant k$ ? It is known that this problem is NP-complete [22, 23, and so is the corresponding problem for global defensive alliances [9]. However, if we restrict ourselves to trees, Defensive Alliance becomes trivial and in fact the corresponding problems for several non-trivial variants become solvable in linear time [22].

There has also been some work on the parameterized complexity of alliance problems. In particular, determining whether a defensive, offensive and powerful alliance of a given (maximum) size exists is fixed-parameter tractable when parameterized by the solution size [16, 15]. Also structural parameters have been considered to some extent. Recently, 24] proved that these problems can be solved in polynomial time if the clique-width of the instances is bounded by a constant. The authors also provide an FPT algorithm when the parameter is the size of the smallest vertex cover. Moreover, [15] showed that the decision problems for defensive alliances and global defensive alliances are fixed-parameter tractable when parameterized by the combination of treewidth and maximum degree. Despite these advances regarding, the question of whether or not Defensive Alliance parameterized by treewidth is fixed-parameter tractable has so far remained open.

Treewidth [30, 5, 7] is one of the most extensively studied structural parameters and indicates how close a graph is to being a tree. It is particularly attractive because many hard problems become tractable on instances of bounded treewidth, and in several practical applications it has been observed that the considered problem instances exhibit small treewidth [5, 33, 26. Hence it would be very appealing to obtain an FPT algorithm for the Defensive Alliance problem using this parameter.

The main contribution of this paper is a parameterized complexity analysis of DefEnsive AlLiance with treewidth as the parameter. The question of whether or not this problem is fixed-parameter tractable when parameterized by treewidth has so far been unresolved [24]. In the current chapter, we provide a negative answer to this question: We show that the problem is hard for the class W[1], which rules out fixed-parameter tractable algorithms under commonly held complexity-theoretic assumptions. This result is rather surprising for two reasons: First, the problem is tractable on trees [22] and quite often problems that become easy on trees turn out to become easy on graphs of bounded treewidth Second, this puts Defensive Alliance among the very few "subset problems" that are fixed-parameter tractable w.r.t. solution size but not w.r.t. treewidth. Problems with this kind of behavior are rather rare, as observed by Dom et al. [13.

We show W[1]-hardness of the problem by first reducing a problem known to be W[1]-hard to a variant of Defensive Alliance, where vertices can be forced to be in or out of every solution, and pairs of vertices can be specified to indicate that every solution must contain exactly one element of each such pair. In order to prove the desired complexity result, we then successively reduce this variant to the standard Defensive AlLiance problem.

1 To be precise, 22, 21, 10, show that some variants of Defensive Alliance are tractable on trees, since Defensive Aldiance on trees is trivial. 
At the same time, we show $\mathrm{W}[1]$-hardness for the exact variants of these problems, where we are interested in defensive alliances exactly of a certain size. Note that a set may lose the property of being a defensive alliance by adding or removing elements, so these are non-trivial problem variants. Indeed, exact versions of alliance problems have also been mentioned as interesting variants in [16] because some algorithms that work for the non-exact case stop to work for the exact case: A graph has a defensive alliance of size at most $k$ if and only if it has a connected defensive alliance of size at most $k$ since every component of a defensive alliance is itself a defensive alliance. Algorithms that exploit this by looking only for connected solutions hence fail for the exact versions. (In fact, we will also study the complexity of other problem variants where this connectedness property does not apply even in the non-exact case.)

This paper is organized as follows: We first introduce our problems of interest and describe preliminary concepts in Section 2 In Section 3 we then show that the Defensive Alliance problem is W[1]-hard when parameterized by treewidth. Section 4 concludes the paper with a discussion.

The reductions in the current work are based on ideas used in the paper [4, which analyzed the complexity of a problem related to Defensive Alliance called Secure Set. That paper has since been extended by a W[1]-hardness proof for the SECURE SET problem parameterized by treewidth [3]. In the current paper, we take up the ideas behind this hardness proof and apply them to the Defensive Alliance problem. Due to the different nature of these two problems, the reductions and proofs for SECURE SET do not work directly for Defensive Alliance but require substantial modifications.

\section{Background}

All graphs are undirected and simple unless stated otherwise. We denote the set of vertices and edges of a graph $G$ by $\mathrm{V}(G)$ and $\mathrm{E}(G)$, respectively. We denote an undirected edge between vertices $u$ and $v$ as $(u, v)$ or equivalently $(v, u)$. It will be clear from the context whether an edge $(u, v)$ is directed or undirected. Given a graph $G$, the open neighborhood of a vertex $v \in \mathrm{V}(G)$, denoted by $N_{G}(v)$, is the set of all vertices adjacent to $v$, and $N_{G}[v]=N_{G}(v) \cup\{v\}$ is called the closed neighborhood of $v$. If it is clear from the context which graph is meant, we write $N(\cdot)$ and $N[\cdot]$ instead of $N_{G}(\cdot)$ and $N_{G}[\cdot]$, respectively.

The intuition behind defensive alliances is the following: If we consider a set $S$ of vertices as "good" vertices and all other vertices as "bad" ones, then $S$ being a defensive alliance means that each element of $S$ has at least as many "good" neighbors as "bad" neighbors.

- Definition 1. Given a graph $G$, a set $S \subseteq \mathrm{V}(G)$ is a defensive alliance in $G$ if for each $v \in S$ it holds that $|N[v] \cap S| \geqslant|N[v] \backslash S|$.

We often write " $S$ is a defensive alliance" instead of " $S$ is a defensive alliance in $G$ " if it is clear from the context which graph is meant. By definition, the empty set is a defensive alliance in any graph. Thus, in the following decision problems we ask for a defensive alliances of size at least 1. For example, in Figure 1 . the set $S=\{a, b\}$ is a defensive alliance as $|N[v] \cap S| \geqslant|N[v] \backslash S|$ holds for each $v \in S$. Note that, for instance, $\{a, d\}$ is no defensive alliance since $d$ is attacked by three vertices but only has the neighbor $a$ to help defend itself.

Next we introduce several variants of Defensive Alliance that we require in our proofs. The problem Defensive Alliance ${ }^{\mathrm{F}}$ generalizes Defensive Alliance by designating some "forbidden" vertices that may never be in any solution. This variant can be formalized as follows: 


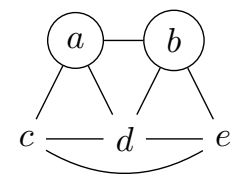

Figure 1 A graph with a minimum non-empty defensive alliance indicated by circled vertices

Defensive Alliance ${ }^{\mathrm{F}}$

Input: A graph $G$, an integer $k$ and a set $V_{\square} \subseteq \mathrm{V}(G)$

Question: Does there exist a set $S \subseteq \mathrm{V}(G) \backslash V_{\square}$ with $1 \leqslant|S| \leqslant k$ that is a defensive alliance?

Defensive AllianCE ${ }^{\mathrm{FN}}$ is a further generalization that, in addition, allows "necessary" vertices to be specified that must occur in every solution.

Defensive Alliance ${ }^{\text {FN }}$

Input: A graph $G$, an integer $k$, a set $V_{\square} \subseteq \mathrm{V}(G)$ and a set $V_{\triangle} \subseteq \mathrm{V}(G)$

Question: Does there exist a set $S \subseteq \mathrm{V}(G) \backslash V_{\square}$ with $V_{\triangle} \subseteq S$ and $1 \leqslant|S| \leqslant k$ that is a defensive alliance?

Finally, we introduce the generalization Defensive Alliance ${ }^{\mathrm{FNC}}$. Here we may state pairs of "complementary" vertices where each solution must contain exactly one element of every such pair.

Defensive Alliance ${ }^{\text {FNC }}$

Input: A graph $G$, an integer $k$, a set $V_{\square} \subseteq \mathrm{V}(G)$, a set $V_{\triangle} \subseteq \mathrm{V}(G)$ and a set $C \subseteq \mathrm{V}(G)^{2}$

Question: Does there exist a set $S \subseteq \mathrm{V}(G) \backslash V_{\square}$ with $V_{\triangle} \subseteq S$ and $1 \leqslant|S| \leqslant k$ that is a defensive alliance and, for each pair $(a, b) \in C$, contains either $a$ or $b$ ?

For our results on the parameter treewidth, we need a way to represent the structure of a DEFENSIVE AlLIANCE ${ }^{\text {FNC }}$ instance by a graph that augments $G$ with the information in $C$ :

- Definition 2. Let $I$ be a Defensive Alliance ${ }^{\text {FNC }}$ instance, let $G$ be the graph in $I$ and let $C$ the set of complementary vertex pairs in $I$. By the primal graph of $I$ we mean the undirected graph $G^{\prime}$ with $\mathrm{V}\left(G^{\prime}\right)=\mathrm{V}(G)$ and $\mathrm{E}\left(G^{\prime}\right)=\mathrm{E}(G) \cup C$.

When we speak of the treewidth of an instance of Defensive Alliance, Defensive Alliance $^{\mathrm{F}}$ or Defensive Alliance ${ }^{\mathrm{FN}}$, we mean the treewidth of the graph in the instance. For an instance of Defensive AlLiAnCE ${ }^{\mathrm{FNC}}$, we mean the treewidth of the primal graph.

While the Defensive Alliance problem asks for defensive alliances of size at most $k$, we also consider the ExACT Defensive AlliAnce problem that concerns defensive alliances of size exactly $k$. Analogously, we also define exact versions of the three generalizations of Defensive Alliance presented above.

In this paper's figures, we often indicate necessary vertices by means of a triangular node shape, and forbidden vertices by means of either a square node shape or a superscript square in the node name. If two vertices are complementary, we often express this in the figures by putting a $\neq \operatorname{sign}$ between them. For example, in Figure 2 , the vertices $b$ and $c$ are complementary and occur in no solution together; $a$ and the "anonymous" vertex adjacent to $c$ are necessary and occur in every solution; $d^{\square}$ and the "anonymous" vertex adjacent to $e$ are 


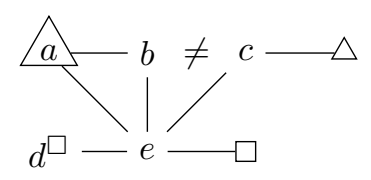

Figure 2 Illustration of forbidden, necessary and complementary vertices

forbidden and occur in no solution. In this figure, the unique minimum non-empty defensive alliance satisfying the conditions of forbidden, necessary and complementary vertices consists of $a, b$ and the "anonymous" necessary vertex adjacent to $c$.

The following terminology will be helpful: We often use the terms attackers and defenders of an element $v$ of a defensive alliance candidate $S$. By these we mean the sets $N[v] \backslash S$ and $N[v] \cap S$, respectively. To show that an element $v$ of a defensive alliance candidate $S$ is not a counterexample to $S$ being a solution, we sometimes employ the notion of a defense of $v$ w.r.t. $S$, which assigns to each attacker a dedicated defender: If we are able to find an injective mapping $\mu: N[v] \backslash S \rightarrow N[v] \cap S$, then obviously $|N[v] \backslash S| \leqslant|N[v] \cap S|$, and we call $\mu$ a defense of $v$ w.r.t. $S$. Given such a defense $\mu$, we say that a defender $d$ repels an attack on $v$ by an attacker $a$ whenever $\mu(a)=d$. Consequentially, when we say that a set of defenders $D$ can repel attacks on $v$ from a set of attackers $A$, we mean that there is a defense that assigns to each element of $A$ a dedicated defender in $D$.

To warm up, we make some easy observations that we will use in our proofs. First, for every set $R$ consisting of a majority of neighbors of a vertex $v$, whenever $v$ is in a defensive alliance, also some element of $R$ must be in it:

Observation 3. Let $S$ be a defensive alliance in a graph, let $v \in S$ and let $R \subseteq N(v)$. If $|R|>\frac{1}{2} N[v]$, then $S$ contains an element of $R$.

Proof. Suppose that $|R|>\frac{1}{2}|N[v]|$ and $S$ contains no element of $R$. Since all elements of $R$ attack $v,|N[v] \backslash S|>\frac{1}{2}|N[v]|$. Hence $2|N[v] \backslash S|>|N[v]|=|N[v] \cap S|+|N[v] \backslash S|$, and we obtain the contradiction $|N[v] \backslash S|>|N[v] \cap S|$.

Next, if one half of the neighbors of an element $v$ of a defensive alliance attacks $v$, then the other half of the neighbors must be in the defensive alliance:

- Observation 4. Let $S$ be a defensive alliance in a graph, let $v \in S$ and let $N(v)$ be partitioned into two equal-sized sets $A, D$. If $A \cap S=\emptyset$, then $D \subseteq S$.

Proof. Since $N(v)$ is partitioned into $A$ and $D$ such that $A \cap S=\emptyset$, we get $N(v) \cap S=D \cap S$. If some element of $D$ is not in $S$, then $D \cap S \subset D$ and $A \subset N[v] \backslash S$. By $|D|=|A|$, we get $|D \cap S|+2 \leqslant|N[v] \backslash S|$. From $|N[v] \cap S|=1+|N(v) \cap S|=1+|D \cap S|$ we now obtain the contradiction $|N[v] \cap S|<|N[v] \backslash S|$.

In particular, if half of the neighbors of $v$ are forbidden, then $v$ can only be in a defensive alliance if all non-forbidden neighbors are also in the defensive alliance.

Finally, we recapitulate some background from complexity theory. In parameterized complexity theory [14, 19, 29, 11, we study problems that consist not only of an input and a question, but also of some parameter of the input that is represented as an integer. A problem is in the class FPT ("fixed-parameter tractable") if it can be solved in time $f(k) \cdot n^{c}$, where $n$ is the input size, $k$ is the parameter, $f$ is a computable function that only depends on $k$, and $c$ is a constant that does not depend on $k$ or $n$. We call such an algorithm an FPT algorithm, and we call it fixed-parameter linear if $c=1$. Similarly, a problem is in the 
class XP ("slice-wise polynomial") if it can be solved in time $f(k) \cdot n^{g(k)}$, where $f$ and $g$ are computable functions. Note that here the degree of the polynomial may depend on $k$, so such algorithms are generally slower than FPT algorithms. For the class W[1] it holds that FPT $\subseteq \mathrm{W}[1] \subseteq \mathrm{XP}$, and it is commonly believed that the inclusions are proper, i.e., W[1]-hard problems do not admit FPT algorithms. W[1]-hardness of a problem can be shown using FPT reductions, which are reductions that run in FPT time and produce an equivalent instance whose parameter is bounded by a function of the original parameter.

For problems whose input can be represented as a graph, one important parameter is treewidth, which is a structural parameter that, roughly speaking, measures the "tree-likeness" of a graph. It is defined by means of tree decompositions, originally introduced in [30. The intuition behind tree decompositions is to obtain a tree from a (potentially cyclic) graph by subsuming multiple vertices under one node and thereby isolating the parts responsible for cyclicity.

- Definition 5. A tree decomposition of a graph $G$ is a pair $\mathcal{T}=(T, \chi)$ where $T$ is a (rooted) tree and $\chi: \mathrm{V}(T) \rightarrow 2^{\mathrm{V}(G)}$ assigns to each node of $T$ a set of vertices of $G$ (called the node's $b a g)$, such that the following conditions are met:

1. For every vertex $v \in \mathrm{V}(G)$, there is a node $t \in \mathrm{V}(T)$ such that $v \in \chi(t)$.

2. For every edge $(u, v) \in \mathrm{E}(G)$, there is a node $t \in \mathrm{V}(T)$ such that $\{u, v\} \subseteq \chi(t)$.

3. For every $v \in \mathrm{V}(G)$, the subtree of $T$ induced by $\{t \in \mathrm{V}(T) \mid v \in \chi(t)\}$ is connected.

We call $\max _{t \in \mathrm{V}(T)}|\chi(t)|-1$ the width of $\mathcal{T}$. The treewidth of a graph is the minimum width over all its tree decompositions.

In general, constructing an optimal tree decomposition (i.e., a tree decomposition with minimum width) is intractable [1. However, the problem is solvable in linear time on graphs of bounded treewidth (specifically in time $w^{\mathcal{O}\left(w^{3}\right)} \cdot n$, where $w$ is the treewidth) [6] and there are also heuristics that offer good performance in practice [12, 8].

In this paper we will consider so-called nice tree decompositions:

- Definition 6. A tree decomposition $\mathcal{T}=(T, \chi)$ is nice if each node $t \in \mathrm{V}(T)$ is of one of the following types:

1. Leaf node: The node $t$ has no child nodes.

2. Introduce node: The node $t$ has exactly one child node $t^{\prime}$ such that $\chi(t) \backslash \chi\left(t^{\prime}\right)$ consists of exactly one element.

3. Forget node: The node $t$ has exactly one child node $t^{\prime}$ such that $\chi\left(t^{\prime}\right) \backslash \chi(t)$ consists of exactly one element.

4. Join node: The node $t$ has exactly two child nodes $t_{1}$ and $t_{2}$ with $\chi(t)=\chi\left(t_{1}\right)=\chi\left(t_{2}\right)$.

Additionally, the bags of the root and the leaves of $T$ are empty.

A tree decomposition of width $w$ for a graph with $n$ vertices can be transformed into a nice one of width $w$ with $\mathcal{O}(w n)$ nodes in fixed-parameter linear time [25].

For any tree decomposition $\mathcal{T}$ and an element $v$ of some bag in $\mathcal{T}$, we use the notation $t_{v}^{\mathcal{T}}$ to denote the unique "topmost node" whose bag contains $v$ (i.e., $t_{v}^{\mathcal{T}}$ does not have a parent whose bag contains $v$ ). Figure 3 depicts a graph and a nice tree decomposition, where we also illustrate the $t_{v}^{\mathcal{T}}$ notation.

When we speak of the treewidth of an instance of Defensive Alliance, Defensive Alliance $^{\mathrm{F}}$, Defensive Alliance ${ }^{\mathrm{FN}}$, Exact Defensive Alliance, Exact Defensive Alliance $^{\mathrm{F}}$ or Exact Defensive Alliance ${ }^{\mathrm{FN}}$, we mean the treewidth of the graph in the instance. For an instance of Defensive Alliance ${ }^{\mathrm{FNC}}$ or Exact Defensive Alliance ${ }^{\mathrm{FNC}}$, we mean the treewidth of the primal graph. 
$G:$<smiles></smiles>

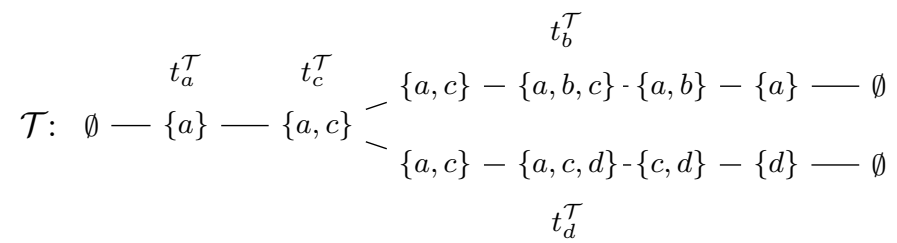

Figure 3 A graph $G$ and a nice tree decomposition $\mathcal{T}$ of $G$ rooted at the leftmost node

\section{Hardness of Defensive Alliance Parameterized by Treewidth}

In this section, we prove the following theorem:

- Theorem 7. The following problems are all $\mathrm{W}[1]$-hard when parameterized by treewidth: Defensive Alliance, Exact Defensive Alliance, Defensive Alliance ${ }^{\mathrm{F}}$, Exact Defensive Alliance $^{\mathrm{F}}$, Defensive Alliance ${ }^{\mathrm{FN}}$, Exact Defensive Alliance ${ }^{\mathrm{FN}}$, DeFensive Alliance ${ }^{\mathrm{FNC}}$, and ExaCt Defensive Alliance ${ }^{\mathrm{FNC}}$.

We prove hardness by providing a chain of FPT reductions from a $\mathrm{W}[1]$-hard problem to the problems under consideration. Under the widely held assumption that FPT $\neq \mathrm{W}[1]$, this rules out fixed-parameter tractable algorithms for these problems.

\subsection{Hardness of Defensive Alliance with Forbidden, Necessary and Complementary Vertices}

To show W[1]-hardness of Defensive Alliance ${ }^{\text {FNC }}$, we reduce from the following problem [2, which is known to be W[1]-hard [32 parameterized by the treewidth of the graph:

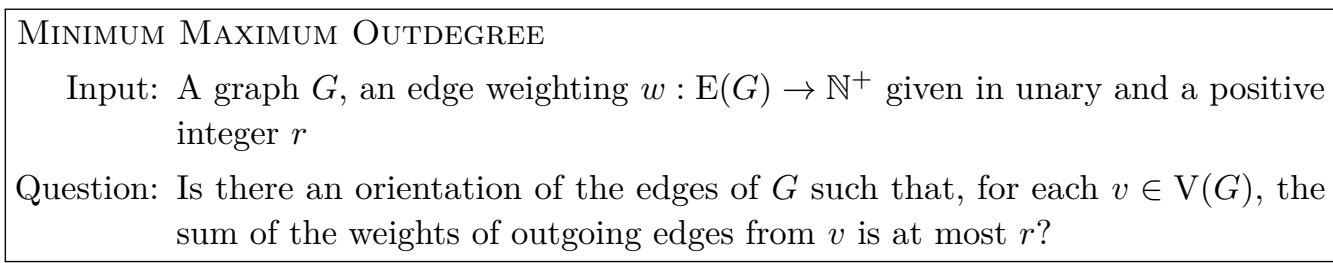

- Lemma 8. Defensive Alliance ${ }^{\mathrm{FNC}}$ and ExACt Defensive Alliance ${ }^{\mathrm{FNC}}$, both parameterized by the treewidth of the primal graph, are $\mathrm{W}[1]$-hard.

Proof. Let an instance of Minimum Maximum Outdegree be given by a graph $G$, an edge weighting $w: \mathrm{E}(G) \rightarrow \mathbb{N}^{+}$in unary and a positive integer $r$. From this we construct an instance of both Defensive Alliance ${ }^{\text {FNC }}$ and Exact Defensive Alliance ${ }^{\text {FNC }}$. An example is given in Figure 4 For each $v \in \mathrm{V}(G)$, we define the set of new vertices $H_{v}=\left\{h_{1}^{v}, \ldots, h_{2 r-1}^{v}\right\}$, and for each $(u, v) \in \mathrm{E}(G)$, we define the sets of new vertices $V_{u v}=\left\{u_{1}^{v}, \ldots, u_{w(u, v)}^{v}\right\}$, $V_{u v}^{\square}=\left\{u_{1}^{v \square}, \ldots, u_{w(u, v)}^{v \square}\right\}, V_{v u}=\left\{v_{1}^{u}, \ldots, v_{w(u, v)}^{u}\right\}$ and $V_{v u}^{\square}=\left\{v_{1}^{u \square}, \ldots, v_{w(u, v)}^{u \square}\right\}$. We now define the graph $G^{\prime}$ with

$$
\begin{aligned}
\mathrm{V}\left(G^{\prime}\right) & =\mathrm{V}(G) \cup \bigcup_{v \in \mathrm{V}(G)} H_{v} \cup \bigcup_{(u, v) \in \mathrm{E}(G)}\left(V_{u v} \cup V_{u v}^{\square} \cup V_{v u} \cup V_{v u}^{\square}\right), \\
\mathrm{E}\left(G^{\prime}\right) & =\left\{(v, h) \mid v \in \mathrm{V}(G), h \in H_{v}\right\} \\
& \cup\left\{(u, x) \mid(u, v) \in \mathrm{E}(G), x \in V_{u v} \cup V_{u v}^{\square}\right\} \\
& \cup\left\{(x, v) \mid(u, v) \in \mathrm{E}(G), x \in V_{v u} \cup V_{v u}^{\square}\right\} .
\end{aligned}
$$




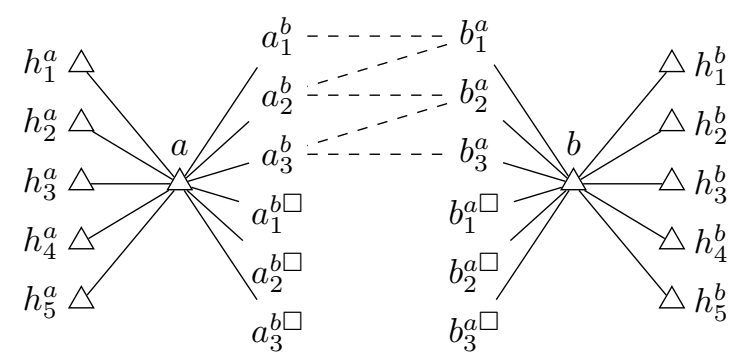

Figure 4 Result of our transformation on a sample Minimum Maximum Outdegree instance with $r=3$ and two vertices $a, b$ that are connected by an edge of weight 3. Complementary vertex pairs are shown via dashed lines. Necessary and forbidden vertices have a $\triangle$ and $\square$ symbol next to their name, respectively.

We also define the set of complementary vertex pairs $C=\left\{\left(u_{i}^{v}, v_{i}^{u}\right) \mid(u, v) \in \mathrm{E}(G), 1 \leqslant i \leqslant\right.$ $w(u, v)\} \cup\left\{\left(v_{i}^{u}, u_{i+1}^{v}\right) \mid(u, v) \in \mathrm{E}(G), 1 \leqslant i<w(u, v)\right\}$. Finally, we define the set of necessary vertices $V_{\triangle}=\mathrm{V}(G) \cup \bigcup_{v \in \mathrm{V}(G)} H_{v}$, the set of forbidden vertices $V_{\square}=\bigcup_{(u, v) \in \mathrm{E}(G)}\left(V_{u v}^{\square} \cup V_{v u}^{\square}\right)$ and $k=\left|V_{\triangle}\right|+\sum_{(u, v) \in \mathrm{E}(G)} w(u, v)$. We use $I$ to denote $\left(G^{\prime}, k, C, V_{\triangle}, V_{\square}\right)$, which is an instance of Defensive Alliance ${ }^{\mathrm{FNC}}$ and also of Exact Defensive Alliance ${ }^{\mathrm{FNC}}$.

Clearly $I$ can be computed in polynomial time. We now show that the treewidth of the primal graph of $I$ depends only on the treewidth of $G$. We do so by modifying an optimal tree decomposition $\mathcal{T}$ of $G$ as follows:

1. For each $(u, v) \in \mathrm{E}(G)$, we take an arbitrary node whose bag $B$ contains both $u$ and $v$ and add to its children a chain of nodes $N_{1}, \ldots, N_{w(u, v)-1}$ such that the bag of $N_{i}$ is $B \cup\left\{u_{i}^{v}, u_{i+1}^{v}, v_{i}^{u}, v_{i+1}^{u}\right\}$.

2. For each $(u, v) \in \mathrm{E}(G)$, we take an arbitrary node whose bag $B$ contains $u$ and add to its children a chain of nodes $N_{1}, \ldots, N_{w(u, v)}$ such that the bag of $N_{i}$ is $B \cup\left\{u_{i}^{v \square}\right\}$.

3. For each $(u, v) \in \mathrm{E}(G)$, we take an arbitrary node whose bag $B$ contains $v$ and add to its children a chain of nodes $N_{1}, \ldots, N_{w(u, v)}$ such that the bag of $N_{i}$ is $B \cup\left\{v_{i}^{u \square}\right\}$.

4. For each $v \in \mathrm{V}(G)$, we take an arbitrary node whose bag $B$ contains $v$ and add to its children a chain of nodes $N_{1}, \ldots, N_{r-1}$ such that the bag of $N_{i}$ is $B \cup\left\{h_{i}^{v}\right\}$.

It is easy to verify that the result is a valid tree decomposition of the primal graph of $I$ and its width is at most the treewidth of $G$ plus four.

It remains to show that our reduction is correct. Obviously $I$ is a positive instance of Defensive Alliance ${ }^{\text {FNC }}$ iff it is a positive instance of ExaCt Defensive Alliance ${ }^{\text {FNC }}$ because the forbidden, necessary and complementary vertices make sure that every solution of the Defensive Alliance ${ }^{\mathrm{FNC}}$ instance $I$ has exactly $k$ elements. Hence we only consider Defensive Alliance ${ }^{\mathrm{FNC}}$.

The intention is that for each orientation of $G$ we have a solution candidate $S$ in $I$ such that an edge orientation from $u$ to $v$ entails $V_{v u} \subseteq S$ and $V_{u v} \cap S=\emptyset$, and the other orientation entails $V_{u v} \subseteq S$ and $V_{v u} \cap S=\emptyset$. For each vertex $v \in \mathrm{V}(G)$ and every incident edge $(v, u) \in \mathrm{E}(G)$ regardless of its orientation, the vertex $v$ is attacked by the forbidden vertices $V_{v u}^{\square}$. So every vertex $v \in \mathrm{V}(G)$ has as least as many attackers as the sum of the weights of all incident edges. If in the orientation of $G$ all edges incident to $v$ are incoming edges, then each attack on $v$ from $V_{v u}^{\square}$ can be repelled by $V_{v u}$, since $V_{v u} \subseteq S$. Due to the fact that the helper vertices $H_{v}$ consist of exactly $2 r-1$ elements, $v$ can afford to have outgoing edges of total weight at most $r$. 
We claim that $(G, w, r)$ is a positive instance of Minimum Maximum Outdegree iff $I$ is a positive instance of Defensive Alliance ${ }^{\mathrm{FNC}}$.

"Only if" direction. Let $D$ be the directed graph given by an orientation of the edges of $G$ such that for each vertex the sum of weights of outgoing edges is at most $r$. The set $S=V_{\triangle} \cup\left\{v_{1}^{u}, \ldots, v_{w(u, v)}^{u} \mid(u, v) \in \mathrm{E}(D)\right\}$ is a defensive alliance in $G^{\prime}$ : Let $x$ be an arbitrary element of $S$. If $x$ is an element of a set $H_{v}$ or $V_{u v}$, then the only neighbor of $x$ in $G^{\prime}$ is a necessary vertex, so $x$ can trivially defend itself; so suppose $x \in \mathrm{V}(G)$. Let the sum of the weights of outgoing and incoming edges be denoted by $w_{\text {out }}^{x}$ and $w_{\text {in }}^{x}$, respectively. The neighbors of $x$ that are also in $S$ consist of the elements of $H_{x}$ and all elements of sets $V_{x v}$ such that $(v, x) \in \mathrm{E}(D)$. Hence, including itself, $x$ has $2 r+w_{\text {in }}^{x}$ defenders in $G^{\prime}$. The attackers of $x$ consist of all elements of sets $V_{x v}$ such that $(x, v) \in \mathrm{E}(D)$ (in total $w_{\text {out }}^{x}$ ) and all elements of sets $V_{x v}^{\square}$ such that either $(v, x) \in \mathrm{E}(D)$ or $(x, v) \in \mathrm{E}(D)$ (in total $w_{\text {in }}^{x}+w_{\text {out }}^{x}$ ). Hence $x$ has $w_{\text {in }}^{x}+2 w_{\text {out }}^{x}$ attackers in $G^{\prime}$. This shows that $x$ has at least as many defenders as attackers, as by assumption $w_{\text {out }}^{x} \leqslant r$. Finally, it is easy to verify that $|S|=k, V_{\square} \cap S=\emptyset$, $V_{\triangle} \subseteq S$, and exactly one element of each pair of complementary vertices is in $S$.

"If" direction. Let $S$ be a solution of $I$. For every $(u, v) \in \mathrm{E}(G)$, either $V_{u v} \subseteq S$ or $V_{v u} \subseteq S$ due to the complementary vertex pairs. We define a directed graph $D$ by $\mathrm{V}(D)=\mathrm{V}(G)$ and $\mathrm{E}(D)=\left\{(u, v) \mid V_{v u} \subseteq S\right\} \cup\left\{(v, u) \mid V_{u v} \subseteq S\right\}$. Suppose there is a vertex $x$ in $D$ whose sum of weights of outgoing edges is greater than $r$. Clearly $x \in S$. Let the sum of the weights of outgoing and incoming edges be denoted by $w_{\text {out }}^{x}$ and $w_{\mathrm{in}}^{x}$, respectively. The defenders of $x$ in $G^{\prime}$ beside itself consist of the elements of $H_{x}$ and of $w_{\text {in }}^{x}$ neighbors due to incoming edges in $D$. These are in total $2 r+w_{\text {in }}^{x}$ defenders. The attackers of $x$ in $G^{\prime}$ consist of $2 w_{\text {out }}^{x}$ elements (of the form $x_{i}^{v}$ as well as $x_{i}^{v \square}$ ) due to outgoing edges in $D$ and $w_{\text {in }}^{x}$ elements (of the form $x_{i}^{v \square}$ ) due to incoming edges. These are in total $2 w_{\text {out }}^{x}+w_{\text {in }}^{x}$ attackers. But then $x$ has more attackers than defenders, as by assumption $w_{\text {out }}^{x}>r$.

\subsection{Hardness of Defensive Alliance with Forbidden and Necessary Vertices}

Next we present a transformation $\tau^{\mathrm{FNC}}$ that eliminates complementary vertex pairs by turning a DefEnsive Alliance ${ }^{\mathrm{FNC}}$ instance into an equivalent DeFENSIVE AlliAnCE ${ }^{\mathrm{FN}}$ instance. Along with $\tau^{\mathrm{FNC}}$, we define a function $\sigma_{I}^{\mathrm{FNC}}$, for each DEFENSIVE ALLIANCE ${ }^{\mathrm{FNC}}$ instance $I$, such that the solutions of $I$ are in a one-to-one correspondence with those of $\tau^{\mathrm{FNC}}(I)$ in such a way that any two solutions of $I$ have the same size iff the corresponding solutions of $\tau^{\mathrm{FNC}}(I)$ have the same size. We use these functions to obtain a polynomial-time reduction from Defensive Alliance ${ }^{\mathrm{FNC}}$ to Defensive Alliance ${ }^{\mathrm{FN}}$ as well as from ExaCT Defensive Alliance ${ }^{\text {FNC }}$ to Exact Defensive Alliance ${ }^{\text {FN }}$.

Before we formally define our reduction, we briefly describe the intuition behind the used gadgets. The gadget in Figure 5 adds neighbors $a_{1}, \ldots, a_{n}, a_{1}^{\square}, \ldots, a_{n}^{\square}$ to every vertex $a$, which are so many that $a$ can only be in a solution if some of the new neighbors are also in the solution. The new vertices are structured in such a way that every solution must in fact either contain all of $a, a_{1}, \ldots, a_{n}$ or none of them. Next, the gadget in Figure 6 is added for every complementary pair $(a, b)$. This gadget is constructed in such a way that every solution must either contain all of $a_{n}, a^{a b}, a_{1}^{a b}, \ldots, a_{n^{2}+n}^{a b}$ or none of them, and the same holds for $b_{n}, b^{a b}, b_{1}^{a b}, \ldots, b_{n^{2}+n}^{a b}$. By making the vertex $\triangle^{a b}$ necessary, every solution must contain one of these two sets. At the same time, the bound on the solution size makes sure that we cannot afford to take both sets for any complementary pair. 


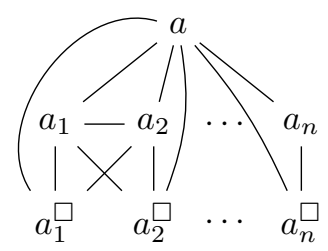

Figure 5 Gadget for each vertex $a$ of the original graph in the reduction from SECURE SET $^{\text {FNC }}$ to SECURE SET ${ }^{\mathrm{FN}}$. The vertex $a$ may have additional neighbors from the original graph, and the vertices $a_{n}$ and $a_{n}^{\square}$ may have additional neighbors as depicted in Figure 6

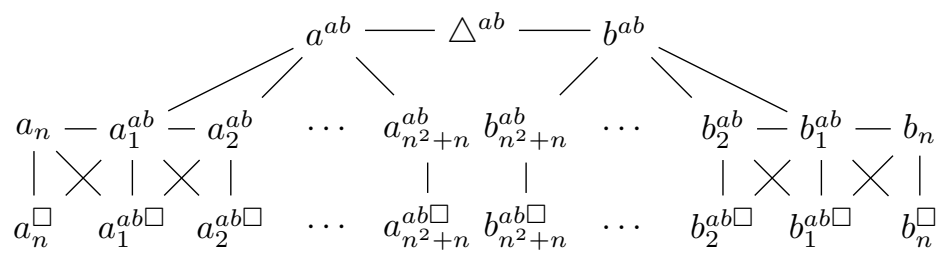

Figure 6 Gadget for each pair of complementary vertices $(a, b)$ in the reduction from SECURE $\operatorname{SET}^{\mathrm{FNC}}$ to SECURE $\mathrm{SET}^{\mathrm{FN}}$. The vertices $a_{n}, a_{n}^{\square}, b_{n}$ and $b_{n}^{\square}$ have additional neighbors as depicted in Figure 5 .

- Definition 9. We define a function $\tau^{\mathrm{FNC}}$, which assigns a Defensive Alliance ${ }^{\mathrm{FN}}$ instance to each Defensive Alliance ${ }^{\mathrm{FNC}}$ instance $I=\left(G, k, V_{\square}, V_{\triangle}, C\right)$. For this, we use $n$ to denote $|\mathrm{V}(G)|$ and first define a function

$$
\sigma_{I}^{\mathrm{FNC}}: x \mapsto x \cdot(n+1)+|C| \cdot\left(n^{2}+n+2\right) .
$$

For each $v \in \mathrm{V}(G)$, we introduce the following sets of new vertices.

$$
Y_{v}^{\circ}=\left\{v_{1}, \ldots, v_{n}\right\} \quad Y_{v}^{\square}=\left\{v_{1}^{\square}, \ldots, v_{n}^{\square}\right\}
$$

Next, for each $(a, b) \in C$, we introduce new vertices $a^{a b}, b^{a b}$ and $\triangle^{a b}$ as well as, for any $x \in\{a, b\}$, the following sets of new vertices.

$$
Z_{x \bigcirc}^{a b}=\left\{x_{1}^{a b}, \ldots, x_{n^{2}+n}^{a b}\right\} \quad Z_{x \square}^{a b}=\left\{x_{1}^{a b \square}, \ldots, x_{n^{2}+n}^{a b \square}\right\}
$$

We use the notation $u \oplus v$ to denote the set of edges $\left\{(u, v),\left(u, u^{\square}\right),\left(v, v^{\square}\right),\left(u, v^{\square}\right),\left(v, u^{\square}\right)\right\}$.

Now we define the Defensive Alliance ${ }^{\mathrm{FN}}$ instance $\tau^{\mathrm{FNC}}(I)=\left(G^{\prime}, k^{\prime}, V_{\square}^{\prime}, V_{\triangle}^{\prime}\right)$, where $k^{\prime}=\sigma_{I}^{\mathrm{FNC}}(k), V_{\square}^{\prime}=V_{\square} \cup \bigcup_{v \in \mathrm{V}(G)} Y_{v}^{\square} \cup \bigcup_{(a, b) \in C}\left(Z_{a \square}^{a b} \cup Z_{b \square}^{a b}\right), V_{\triangle}^{\prime}=V_{\triangle} \cup \bigcup_{(a, b) \in C}\left\{\triangle^{a b}\right\}$ and $G^{\prime}$ is the graph defined by

$$
\begin{gathered}
\mathrm{V}\left(G^{\prime}\right)=\mathrm{V}(G) \cup \bigcup_{v \in \mathrm{V}(G)}\left(Y_{v}^{\bigcirc} \cup Y_{v}^{\square}\right) \cup \\
\cup \bigcup_{(a, b) \in C}\left(\left\{\triangle^{a b}, a^{a b}, b^{a b}\right\} \cup Z_{a \bigcirc}^{a b} \cup Z_{b \bigcirc}^{a b} \cup Z_{a \square}^{a b} \cup Z_{b \square}^{a b}\right), \\
\mathrm{E}\left(G^{\prime}\right)=\mathrm{E}(G) \cup \bigcup_{v \in \mathrm{V}(G)}\left(\left(\{v\} \times Y_{v}^{\circ}\right) \cup\left(\{v\} \times Y_{v}^{\square}\right) \cup \bigcup_{1 \leqslant i<n} v_{i} \oplus v_{i+1}\right) \cup \\
\cup \bigcup_{(a, b) \in C} \bigcup_{x \in\{a, b\}}\left(\left\{\left(\triangle^{a b}, x^{a b}\right)\right\} \cup\left(\left\{x^{a b}\right\} \times Z_{x \bigcirc}^{a b}\right) \cup\right. \\
\left.\cup x_{n} \oplus x_{1}^{a b} \cup \bigcup_{1 \leqslant i<n^{2}+n} x_{i}^{a b} \oplus x_{i+1}^{a b}\right) .
\end{gathered}
$$


We illustrate our construction in Figures 5 and 6

- Lemma 10. Let $I=\left(G, k, V_{\square}, V_{\triangle}, C\right)$ be a Defensive Alliance ${ }^{\mathrm{FNC}}$ instance, let $A$ be the set of solutions of $I$ and let $B$ be the set of solutions of the DEFENSIVE ALLIANCE ${ }^{\mathrm{FN}}$ instance $\tau^{F N C}(I)$. There is a bijection $f: A \rightarrow B$ such that $|f(S)|=\sigma_{I}^{F N C}(|S|)$ holds for every $S \in A$.

Proof. We use the same auxiliary notation as in Definition 9 and we define $f$ as $S \mapsto$ $S \cup \bigcup_{v \in S} Y_{v}^{\bigcirc} \cup \bigcup_{(a, b) \in C, x \in S \cap\{a, b\}}\left(\left\{\triangle^{a b}, x^{a b}\right\} \cup Z_{x \circ}^{a b}\right)$. For every $S \in A$, we thus obtain $|f(S)|=\sigma_{I}^{\mathrm{FNC}}(|S|)$, and we first show that indeed $f(S) \in B$.

Let $S \in A$ and let $S^{\prime}$ denote $f(S)$. Obviously $S^{\prime}$ satisfies $V_{\square}^{\prime} \cap S^{\prime}=\emptyset$ and $V_{\triangle}^{\prime} \subseteq S^{\prime}$. To see that $S^{\prime}$ is a defensive alliance in $G^{\prime}$, let $x$ be an arbitrary element of $S^{\prime}$. If $x \notin S$, then $x$ clearly has as least as many neighbors in $S^{\prime}$ as neighbors not in $S^{\prime}$ by construction of $f$, so suppose $x \in S$. There is a defense $\mu: N_{G}[x] \backslash S \rightarrow N_{G}[x] \cap S$ since $S$ is a defensive alliance in $G$. We use this to construct a defense $\mu^{\prime}: N_{G^{\prime}}[x] \backslash S^{\prime} \rightarrow N_{G^{\prime}}[x] \cap S^{\prime}$. For any attacker $v$ of $x$ in $G^{\prime}$, we distinguish two cases.

- If $v$ is some $x_{i}^{\square} \in Y_{x}^{\square}$ for some $x \in \mathrm{V}(G)$, we set $\mu^{\prime}(v)=x_{i}$. This element is in $N_{G^{\prime}}[x]$ by construction.

- Otherwise $v$ is in $N_{G}[x] \backslash S$ (by our construction of $S^{\prime}$ ). Since the codomain of $\mu$ is a subset of the codomain of $\mu^{\prime}$, we may set $\mu^{\prime}(v)=\mu(v)$.

Since $\mu^{\prime}$ is injective, each attack on $x$ in $G^{\prime}$ can be repelled by $S^{\prime}$. Hence $S^{\prime}$ is a defensive alliance in $G^{\prime}$.

Clearly $f$ is injective. It remains to show that $f$ is surjective. Let $S^{\prime}$ be a solution of $\tau^{\mathrm{FNC}}(I)$. First we make the following observations for each $v \in \mathrm{V}(G)$ :

- If $v \in S^{\prime}$, then $Y_{v}^{\circ} \cap S^{\prime} \neq \emptyset$ due to Observation 3 , since $Y_{v}^{\circ} \cup Y_{v}^{\square}$ contains a majority of neighbors of $v$, and the vertices in $Y_{v}^{\square}$ are forbidden.

- For each $v^{a b} \in S^{\prime}$, where $(a, b) \in C$ such that $v=a$ or $v=b$, it holds that $Z_{v \circ}^{a b} \cap S^{\prime} \neq \emptyset$ again due to Observation 3

- If $S^{\prime}$ contains an element of $Y_{v}^{\circ}$, then $\{v\} \cup Y_{v}^{\circ} \cup \bigcup_{(v, z) \in C} Z_{v \bigcirc}^{v z} \cup \bigcup_{(z, v) \in C} Z_{v \bigcirc}^{z v} \subseteq S^{\prime}$ by repeated applications of Observation 4 To see this, note in particular that $N\left(v_{n}\right)$ can be partitioned into the two equal-sized sets $\left\{v, v_{n-1}\right\} \cup\left\{v_{1}^{v z} \mid(v, z) \in C\right\} \cup\left\{v_{1}^{z v} \mid(z, v) \in C\right\}$ and $\left\{v_{n-1}^{\square}, v_{n}^{\square}\right\} \cup\left\{v_{1}^{v z \square} \mid(v, z) \in C\right\} \cup\left\{v_{1}^{z v \square} \mid(z, v) \in C\right\}$, and all vertices in the latter set are forbidden.

- If $S^{\prime}$ contains an element of $Z_{v \circ}^{a b}$, where $(a, b) \in C$ such that $v=a$ or $v=b$, then $\left\{v^{a b}\right\} \cup Y_{v}^{\bigcirc} \cup \bigcup_{(v, z) \in C} Z_{v \circ}^{v z} \cup \bigcup_{(z, v) \in C} Z_{v \circ}^{z v} \subseteq S^{\prime}$ for similar reasons.

It follows that for each $v \in \mathrm{V}(G), S^{\prime}$ contains either all or none of $\{v\} \cup Y_{v}^{\circ} \cup \bigcup_{(v, z) \in C}\left(\left\{v^{v z}\right\} \cup\right.$ $Z_{v \mathrm{O}}^{v z} \cup \bigcup_{(z, v) \in C}\left(\left\{v^{z v}\right\} \cup Z_{v \bigcirc}^{z v}\right)$.

For every $(a, b) \in C, S^{\prime}$ contains $a^{a b}$ or $b^{a b}$, since $\triangle^{a b} \in S^{\prime}$, whose neighbors are $a^{a b}$ and $b^{a b}$. It follows that $\left|S^{\prime}\right|>|C| \cdot\left(n^{2}+n+2\right)$ even if $S^{\prime}$ contains only one of each $(a, b) \in C$. If, for some $(a, b) \in C, S^{\prime}$ contained both $a$ and $b$, we could derive a contradiction to $\left|S^{\prime}\right| \leqslant$ $\sigma_{I}^{\mathrm{FNC}}(k)=k \cdot(n+1)+|C| \cdot\left(n^{2}+n+2\right)$ because then $\left|S^{\prime}\right|>(|C|+1) \cdot\left(n^{2}+n+2\right)>\sigma_{I}^{\mathrm{FNC}}(k)$. So $S^{\prime}$ contains either $a$ or $b$ for any $(a, b) \in C$.

We construct $S=S^{\prime} \cap \mathrm{V}(G)$ and observe that $S^{\prime}=f(S), V_{\triangle} \subseteq S, V_{\square} \cap S=\emptyset$, and $|S \cap\{a, b\}|=1$ for each $(a, b) \in C$. It remains to show that $S$ is a defensive alliance in $G$. Let $x$ be an arbitrary element of $S$. We observe that $N_{G^{\prime}}[x] \cap S^{\prime}=\left(N_{G}[x] \cap S\right) \cup Y_{x}^{\circ}$ and similarly $N_{G^{\prime}}[x] \backslash S^{\prime}=\left(N_{G}[x] \backslash S\right) \cup Y_{x}^{\square}$. Since the cardinality of each set $Y_{x}^{\circ}$ is equal to the cardinality of $Y_{x}^{\square}$, this implies $\left|N_{G^{\prime}}[x] \cap S^{\prime}\right|-\left|N_{G}[x] \cap S\right|=\left|N_{G^{\prime}}[x] \backslash S^{\prime}\right|-\left|N_{G}[x] \backslash S\right|$. 
Since $S^{\prime}$ is a defensive alliance in $G^{\prime}$ and $x \in S^{\prime}$, it holds that $\left|N_{G^{\prime}}[x] \cap S^{\prime}\right| \geqslant\left|N_{G^{\prime}}[x] \backslash S^{\prime}\right|$. We conclude that $\left|N_{G}[x] \cap S\right| \geqslant\left|N_{G}[x] \backslash S\right|$. Hence $S$ is a defensive alliance in $G$.

To obtain the hardness result for Defensive Alliance ${ }^{\mathrm{FN}}$ parameterized by treewidth, it remains to show that the reduction specified by $\tau^{\mathrm{FNC}}$ preserves bounded treewidth.

- Lemma 11. Defensive Alliance ${ }^{\mathrm{FN}}$, parameterized by the treewidth of the graph, is W[1]-hard.

Proof. Let $I$ be a Defensive Alliance ${ }^{\mathrm{FNC}}$ instance whose primal graph we denote by $G$. We obtain an equivalent Defensive Alliance ${ }^{\mathrm{FN}}$ instance $\tau^{\mathrm{FNC}}(I)$, whose graph we denote by $G^{\prime}$. This reduction is correct, as shown in Lemma 10. It remains to show that the treewidth of $G^{\prime}$ is bounded by a function of the treewidth of $G$. Let $\mathcal{T}$ be an optimal nice tree decomposition of $G$. We build a tree decomposition $\mathcal{T}^{\prime}$ of $G^{\prime}$ by modifying a copy of $\mathcal{T}$ in the following way: For each vertex $v \in \mathrm{V}(G)$, we add $v_{n}$ and $v_{n}^{\square}$ to every bag containing $v$. Then we pick an arbitrary node $t$ in $\mathcal{T}$ whose bag contains $v$, and we add new children $N_{1}, \ldots, N_{n-1}$ to $t$ such that the bag of $N_{i}$ is $\left\{v, v_{i}, v_{i}^{\square}, v_{i+1}, v_{i+1}^{\square}\right\}$. Next, for every pair $(a, b)$ of complementary vertices, we pick an arbitrary node $t$ in $\mathcal{T}$ whose bag $B$ contains both $a_{n}$ and $b_{n}$, and we add a chain of nodes $N_{1}, \ldots, N_{2 n^{2}+2 n-1}$ between $t$ and its parent such that, for $1 \leqslant i<$ $n^{2}+n$, the bag of $N_{i}$ is $B \cup\left\{a^{a b}, a_{i}^{a b}, a_{i}^{a b \square}, a_{i+1}^{a b}, a_{i+1}^{a b \square}\right\}$, the bag of $N_{n^{2}+n}$ is $B \cup\left\{a^{a b}, b^{a b}, \triangle^{a b}\right\}$, and the bag of $N_{n^{2}+n+i}$ is $B \cup\left\{b^{a b}, b_{n^{2}+n+1-i}^{a b}, b_{n^{2}+n+1-i}^{a b \square}, b_{n^{2}+n-i}^{a b}, b_{n^{2}+n-i}^{a b \square}\right\}$. It is easy to verify that $\mathcal{T}^{\prime}$ is a valid tree decomposition of $G^{\prime}$. Furthermore, the width of $\mathcal{T}^{\prime}$ is at most three times the width of $\mathcal{T}$ plus five.

The instances of Defensive Alliance ${ }^{\mathrm{FNC}}$ are identical to the instances of the exact variant, so $\tau^{\mathrm{FNC}}$ is also applicable to the exact case. In fact it turns out that this gives us also a reduction from Exact Defensive Alliance ${ }^{\mathrm{FNC}}$ to Exact Defensive Alliance ${ }^{\mathrm{FN}}$.

- Lemma 12. ExaCt Defensive Alliance ${ }^{\mathrm{FN}}$, parameterized by the treewidth of the graph, is $\mathrm{W}[1]$-hard.

Proof. Let $I$ and $I^{\prime}=\tau^{\mathrm{FNC}}(I)$ be our ExaCt Defensive Alliance ${ }^{\mathrm{FNC}}$ and ExaCT Defensive Alliance ${ }^{\mathrm{FN}}$ instances, respectively, and let $k$ and $k^{\prime}$ denote their respective solution sizes. By Lemma 10, there is a bijection $f$ between the solutions of $I$ and the solutions of $I^{\prime}$ such that, for every solution $S$ of $I, f(S)$ has $\sigma_{I}^{\mathrm{FNC}}(k)=k^{\prime}$ elements, and for every solution $S^{\prime}$ of $I^{\prime}, f^{-1}\left(S^{\prime}\right)$ has $k$ elements since $\sigma_{I}^{\mathrm{FNC}}$ is invertible. We can derive the bound on the treewidth of $I^{\prime}$ as in the proof of Lemma 11

\subsection{Hardness of Defensive Alliance with Forbidden Vertices}

Now we present a transformation $\tau^{\mathrm{FN}}$ that eliminates necessary vertices. Our transformation not only operates on a problem instance, but also requires an ordering $\preceq$ of the non-forbidden vertices of the graph. Our reductions based on $\tau^{\mathrm{FN}}$ will be correct for every ordering $\preceq$, but in order to keep the treewidth of the resulting problem instance bounded by the treewidth of the original problem instance, we must choose a suitable ordering. We will describe this in detail later; for now we can consider $\preceq$ to be an arbitrary ordering of the non-forbidden vertices.

Before formally defining the transformation $\tau^{\mathrm{FN}}$, we refer to Figure 7 which shows the result for a simple example graph with only two vertices $a$ and $b$, of which $b$ is necessary. The basic idea is that the vertex $a^{\prime}$ must be in every solution $S$ : If $a$ or any vertex to the left of $a$ is in $S$, it eventually forces $a^{\prime}$ to be in $S$ as well. Likewise, if $b$ or any vertex to the right of 


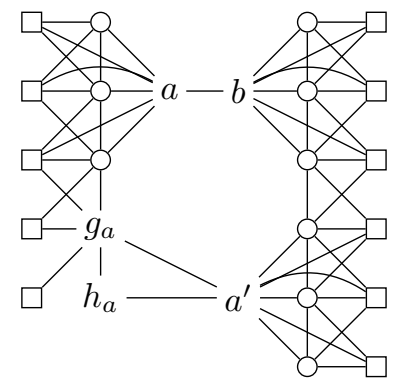

Figure 7 Result of the transformation $\tau^{\mathrm{FN}}$ applied to an example graph with two adjacent vertices $a$ and $b$, where $b$ is necessary. Every solution in the depicted graph contains $a^{\prime}, h_{a}$ and $b$.

$b$ is in $S$, it also forces $a^{\prime}$ to be in $S$. Once $a^{\prime} \in S$, the construction to the right of $a^{\prime}$ makes sure that $b \in S$. We will generalize this to instances containing more vertices so that every necessary vertex as well as the primed copy of each non-necessary vertex is in every solution.

- Definition 13. We define a function $\tau^{\mathrm{FN}}$, which assigns a Defensive Alliance ${ }^{\mathrm{F}}$ instance to each pair $(I, \preceq)$, where $I=\left(G, k, V_{\square}, V_{\triangle}\right)$ is a Defensive Alliance ${ }^{\mathrm{FN}}$ instance and $\preceq$ is an ordering of the non-forbidden elements of $\mathrm{V}(G)$. For this, let $V_{\circ}$ denote $\mathrm{V}(G) \backslash\left(V_{\square} \cup V_{\triangle}\right)$. We use $n$ to denote $|\mathrm{V}(G)|$, and we first define a function $\sigma_{I}^{\mathrm{FN}}: x \mapsto(n+3) \cdot\left(x+\left|V_{0}\right|\right)-\left|V_{\triangle}\right|$. We use $H$ to denote the set of new vertices $\left\{v^{\prime}, g_{v}, h_{v}, g_{v}^{\square}, h_{v}^{\square} \mid v \in V_{0}\right\}$. The intention is for each $g_{v}^{\square}$ and $h_{v}^{\square}$ to be forbidden, for each $v^{\prime}$ and $h_{v}$ to be in every solution, and for $g_{v}$ to be in a solution iff $v$ is in it at the same time. We write $V^{+}$to denote $V_{\triangle} \cup V_{\circ} \cup\left\{v^{\prime} \mid v \in V_{\circ}\right\}$; for each $v \in V^{+}$, we use $A_{v}$ to denote the set of new vertices $\left\{v_{1}, \ldots, v_{n+1}, v_{1}^{\square}, \ldots, v_{n+1}^{\square}\right\}$, and we use shorthand notation $A_{v}^{\circ}=\left\{v_{1}, \ldots, v_{n+1}\right\}$ and $A_{v}^{\square}=\left\{v_{1}^{\square}, \ldots, v_{n+1}^{\square}\right\}$. The intention is for each $v_{i}^{\square}$ to be forbidden and for each $v_{i}$ to be in a solution iff $v$ is in it at the same time. We use the notation $u \oplus v$ to denote the set of edges $\left\{(u, v),\left(u, u^{\square}\right),\left(v, v^{\square}\right),\left(u, v^{\square}\right),\left(v, u^{\square}\right)\right\}$. For any vertex $v \in V_{\odot} \cup V_{\triangle}$, we define $p(v)=v$ if $v \in V_{\triangle}$ and $p(v)=v^{\prime}$ if $v \in V_{\circ}$. Let $P$ be the set consisting of all pairs $(p(u), p(v))$ such that $v$ is the direct successor of $u$ according to $\preceq$. Now we define $\tau^{\mathrm{FN}}(I, \preceq)=\left(G^{\prime}, k^{\prime}, V_{\square}^{\prime}\right)$, where $V_{\square}^{\prime}=V_{\square} \cup\left\{g_{v}^{\square}, h_{v}^{\square} \mid v \in V_{\circ}\right\} \cup \bigcup_{v \in V^{+}} A_{v}^{\square}$, $k^{\prime}=\sigma_{I}^{\mathrm{FN}}(k)$, and $G^{\prime}$ is the graph defined by

$$
\begin{aligned}
\mathrm{V}\left(G^{\prime}\right) & =\mathrm{V}(G) \cup H \cup \bigcup_{v \in V^{+}} A_{v}, \\
\mathrm{E}\left(G^{\prime}\right) & =\mathrm{E}(G) \cup\left\{\left(v, v_{i}\right),\left(v, v_{i}^{\square}\right) \mid v \in V^{+}, 1 \leqslant i \leqslant n+1\right\} \\
& \cup \bigcup_{v \in V^{+}, 1 \leqslant i \leqslant n} v_{i} \oplus v_{i+1} \cup \bigcup_{(u, v) \in P} u_{n+1} \oplus v_{1} \\
& \cup \bigcup_{v \in V_{0}} v_{n+1} \oplus g_{v} \cup\left\{\left(v^{\prime}, g_{v}\right),\left(v^{\prime}, h_{v}\right),\left(g_{v}, h_{v}\right),\left(g_{v}, h_{v}^{\square}\right) \mid v \in V_{0}\right\} .
\end{aligned}
$$

We illustrate our construction in Figure 8 and 9

We now prove that $\tau^{\mathrm{FN}}$ yields a correct reduction for any ordering $\preceq$.

- Lemma 14. Let $I=\left(G, k, V_{\square}, V_{\triangle}\right)$ be a Defensive AlliANCE ${ }^{\mathrm{FN}}$ instance, let $\preceq$ be an ordering of $\mathrm{V}(G) \backslash V_{\square}$, let $A$ be the set of solutions of $I$ and let $B$ be the set of solutions of the Defensive Alliance ${ }^{\mathrm{F}}$ instance $\tau^{F N}(I, \preceq)$. There is a bijection $f: A \rightarrow B$ such that $|f(S)|=\sigma_{I}^{F N}(|S|)$ holds for every $S \in A$. 


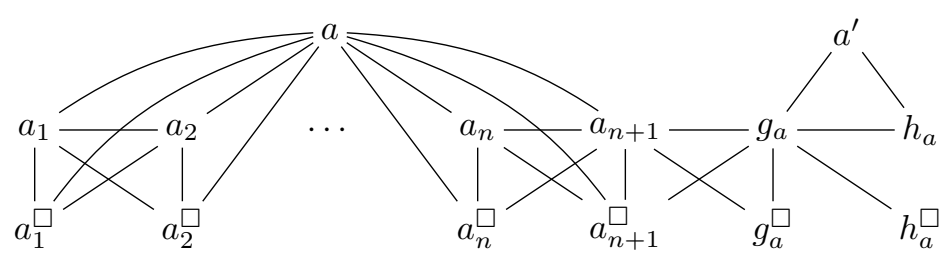

Figure 8 Illustration of the gadget that makes sure that every solution containing $a$ also contains $f_{a}, g_{a}$ and $a^{\prime}$. The vertex $a$ is a non-necessary, non-forbidden vertex from the SECURE SET ${ }^{\mathrm{FN}}$ instance and may have other neighbors from this instance. The vertex $a^{\prime}$ additionally has the neighbors depicted in Figure 9

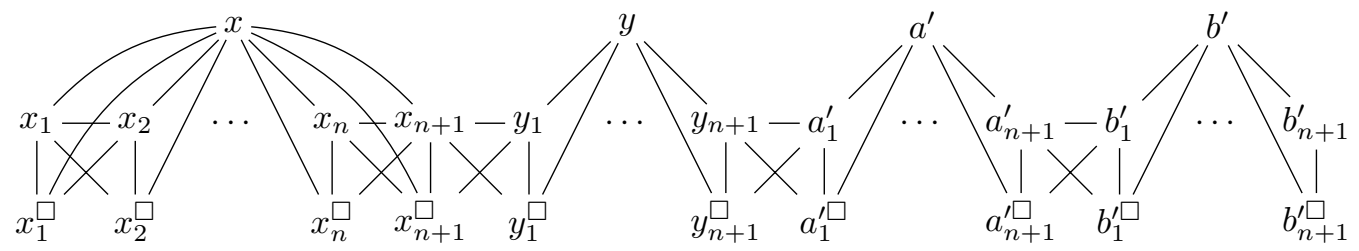

Figure 9 Illustration of the gadget that makes sure that every solution contains all necessary vertices if it contains some necessary vertex or if it contains $v^{\prime}$ for some non-necessary vertex $v$. Here we assume there are the four vertices $a, b, x, y$, among which $x$ and $y$ are necessary, and we use the ordering $x \preceq y \preceq a \preceq b$.

Proof. We use the same auxiliary notation as in Definition 13 and we define $f$ as

$$
f(S)=S \cup \bigcup_{v \in S} A_{v}^{\circ} \cup\left\{v^{\prime}, h_{v} \mid v \in V_{\circ}\right\} \cup \bigcup_{v \in V_{\circ}} A_{v^{\prime}}^{\circ} \cup\left\{g_{v} \mid v \in S \cap V_{\circ}\right\} .
$$

For every $S \in A$, we thus obtain $|f(S)|=|S|+|S|(n+1)+2\left|V_{\circ}\right|+\left|V_{\circ}\right| \cdot(n+1)+\left(|S|-\left|V_{\triangle}\right|\right)=$ $\sigma_{I}^{\mathrm{FN}}(|S|)$, and we first show that indeed $f(S) \in B$.

Let $S \in A$ and let $S^{\prime}$ denote $f(S)$. Obviously $S^{\prime}$ satisfies $V_{\square}^{\prime} \cap S^{\prime}=\emptyset$. To see that $S^{\prime}$ is a defensive alliance in $G^{\prime}$, let $x$ be an arbitrary element of $S^{\prime}$. If $x \in S$, then there is a defense $\mu: N_{G}[x] \backslash S \rightarrow N_{G}[x] \cap S$ since $S$ is a defensive alliance in $G$. We use this to construct a defense $\mu^{\prime}: N_{G^{\prime}}[x] \backslash S^{\prime} \rightarrow N_{G^{\prime}}[x] \cap S^{\prime}$. For any attacker $a$ of $x$ in $G^{\prime}$, we distinguish the following cases:

- If $a$ is some $v_{i}^{\square} \in A_{v}^{\square}$ for some $v \in V^{+}$, then $x$ is either $v_{i}$ or a neighbor of $v_{i}$, all of which are in $S^{\prime}$, and we set $\mu^{\prime}(a)=v_{i}$.

- Similarly, if $a$ is $g_{v}^{\square}$ for some $v \in V_{\circ}$, then we set $\mu^{\prime}(a)=g_{v}$.

- If $a$ is $h_{v}^{\square}$ for some $v \in V_{0}$, then $x=g_{v}$ and we set $\mu^{\prime}(a)=h_{v}$.

- If $a$ is $g_{v}$ for some $v \in V_{\circ}$, then $x$ is either $v^{\prime}$ or $h_{v}$, which is not used for repelling any other attack because $h_{v}^{\square}$ cannot attack $x$, so we set $\mu^{\prime}(a)=h_{v}$.

- Otherwise $a$ is in $N_{G}[x] \backslash S$ (by our construction of $S^{\prime}$ ). Since the codomain of $\mu$ is a subset of the codomain of $\mu^{\prime}$, we may set $\mu^{\prime}(a)=\mu(a)$.

Since $\mu^{\prime}$ is injective, each attack on $x$ in $G^{\prime}$ can be repelled by $S^{\prime}$. Hence $S^{\prime}$ is a defensive alliance in $G^{\prime}$.

Clearly $f$ is injective. It remains to show that $f$ is surjective. Let $S^{\prime}$ be a solution of $\tau^{\mathrm{FN}}(I, \preceq)$. We first show that $V_{\triangle} \cup\left\{v^{\prime}, h_{v} \mid v \in V_{\circ}\right\} \subseteq S^{\prime}$ :

- If $S^{\prime}$ contains some $v \in V^{+}$, then $S^{\prime}$ contains an element of $A_{v}^{\circ}$ by Observation 3 .

- If $S^{\prime}$ contains an element of $A_{v}^{\circ}$ for some $v \in V^{+}$, then $\{v\} \cup A_{v}^{\circ} \subseteq S^{\prime}$ by Observation 4 
- If $v_{n+1} \in S^{\prime}$ for some $v \in V_{0}$, then $g_{v} \in S^{\prime}$ for the same reason.

- Furthermore, if $S^{\prime}$ contains an element of $A_{v}^{\circ}$ for some $v \in V_{\triangle} \cup\left\{v^{\prime} \mid v \in V_{\circ}\right\}$, then also $A_{u}^{\circ} \subseteq S^{\prime}$ for every $u \in V_{\triangle} \cup\left\{v^{\prime} \mid v \in V_{\circ}\right\}$ for the same reason.

- If $g_{v} \in S^{\prime}$ for some $v \in V_{0}$, then $\left\{h_{v}, v^{\prime}, v_{n+1}\right\} \subseteq S^{\prime}$ by Observation 4

- If $h_{v} \in S^{\prime}$ for some $v \in V_{0}$, then $a^{\prime} \in S^{\prime}$ because at least $g_{v}$ or $v^{\prime}$ must be in $S^{\prime}$ and the former implies $v^{\prime} \in S^{\prime}$ as we have seen.

- Since $S^{\prime}$ is nonempty, the previous observations show that for every $v \in V_{\triangle} \cup\left\{v^{\prime} \mid v \in V_{0}\right\}$ it holds that $\{v\} \cup A_{v}^{\circ} \subseteq S^{\prime}$. Finally, we show that $\left\{h_{v} \mid v \in V_{\circ}\right\} \subseteq S^{\prime}$. Suppose, for the sake of contradiction, that there is some $v \in V_{\circ}$ such that $h_{v} \notin S^{\prime}$. We have seen that the latter can only be the case if $g_{v} \notin S^{\prime}$, and we know that $v^{\prime} \in S^{\prime}$. We obtain the contradiction that $v^{\prime}$ is attacked by $g_{v}, h_{v}$ and $A_{v^{\prime}}^{\square}$, whereas its only defenders are $v^{\prime}$ itself and $A_{v^{\prime}}^{\circ}$.

Let $S=S^{\prime} \cap \mathrm{V}(G)$. By the previous observations, it is easy to see that $S^{\prime}=f(S)$. It remains to show that $S$ is a defensive alliance in $G$. Let $x$ be an arbitrary element of $S$. We observe that $N_{G^{\prime}}[x] \cap S^{\prime}=\left(N_{G}[x] \cap S\right) \cup A_{x}^{\bigcirc}$ and similarly $N_{G^{\prime}}[x] \backslash S^{\prime}=\left(N_{G}[x] \backslash S\right) \cup A_{v}^{\square}$. Since $\left|A_{x}^{\bigcirc}\right|=\left|A_{x}^{\square}\right|$, this implies $\left|N_{G^{\prime}}[x] \cap S^{\prime}\right|-\left|N_{G}[x] \cap S\right|=\left|N_{G^{\prime}}[x] \backslash S^{\prime}\right|-\left|N_{G}[x] \backslash S\right|$. Since $S^{\prime}$ is a defensive alliance in $G^{\prime}$ and $x \in S^{\prime}$, it holds that $\left|N_{G^{\prime}}[x] \cap S^{\prime}\right| \geqslant\left|N_{G^{\prime}}[x] \backslash S^{\prime}\right|$. We conclude that $\left|N_{G}[x] \cap S\right| \geqslant\left|N_{G}[x] \backslash S\right|$. Hence $S$ is a defensive alliance in $G$.

Given an ordering $\preceq$, clearly $\tau^{\mathrm{FN}}(I, \preceq)$ is computable in polynomial time. We can thus easily obtain a reduction from Defensive Alliance ${ }^{\mathrm{FN}}$ to Defensive Alliance ${ }^{\mathrm{F}}$ by first computing an arbitrary ordering $\preceq$ of the non-forbidden vertices. We next show that by choosing $\preceq$ appropriately, this amounts to an FPT reduction that preserves bounded treewidth.

- Lemma 15. Defensive Alliance ${ }^{\mathrm{F}}$, parameterized by the treewidth of the graph, is W[1]-hard.

Proof. Let $I=\left(G, k, V_{\square}, V_{\triangle}\right)$ be a Defensive Alliance $^{\mathrm{FN}}$ instance and let $\mathcal{T}$ be an optimal nice tree decomposition of $G$. We can compute such a tree decomposition in FPT time [6]. Let $\preceq$ be the ordering of the elements of $V_{\triangle} \cup V_{\circ}$ that is obtained in linear time by doing a post-order traversal of $\mathcal{T}$ and sequentially recording the elements that occur for the last time in the current bag. We obtain the Defensive Alliance ${ }^{\mathrm{F}}$ instance $\tau^{\mathrm{FN}}(I, \preceq)$, whose graph we denote by $G^{\prime}$. This reduction is correct, as shown in Lemma 14 and computable in FPT time. It remains to show that the treewidth of $G^{\prime}$ is bounded by a function of the treewidth of $G$. To this end, we use $\mathcal{T}$ to build a tree decomposition $\mathcal{T}^{\prime}$ of $G^{\prime}$. We initially set $\mathcal{T}^{\prime}:=\mathcal{T}$ and modify it by the following steps:

1. For each $v \in V_{\circ}$, we add $g_{v}, g_{v}^{\square}, h_{v}, h_{v}^{\square}$ and $v^{\prime}$ to the bag of $t_{v}^{\mathcal{T}^{\prime}}$. Note that afterwards $t_{v}^{\mathcal{T}^{\prime}}=t_{v^{\prime}}^{\mathcal{T}^{\prime}}$. After this step we increased the width of $\mathcal{T}^{\prime}$ by at most five.

2. For each $v \in V^{+}$, we use $B_{v}$ to denote the bag of $t_{v}^{\mathcal{T}^{\prime}}$ and replace $t_{v}^{\mathcal{T}^{\prime}}$ by a chain of nodes $N_{1}, \ldots, N_{n}$, where $N_{n}$ is the topmost node and the bag of $N_{i}$ is $B_{v} \cup\left\{v_{i}, v_{i}^{\square}, v_{i+1}, v_{i+1}^{\square}\right\}$. After this step we increased the width of $\mathcal{T}^{\prime}$ by at most nine. Note that the bag of the new node $t_{v}^{\mathcal{T}^{\prime}}$ now contains $v_{i+1}$ and $v_{i+1}^{\square}$. We have so far covered all edges except the ones connecting elements of two different sets $A_{x}$ and $A_{y}$ for $(x, y) \in P$.

3. For every $(u, v) \in P$, we add $v_{1}$ and $v_{1}^{\square}$ into the bag of every node between (and including) $t_{u}^{\mathcal{T}^{\prime}}$ and $t_{v_{1}}^{\mathcal{T}^{\prime}}$. Note that this preserves connectedness and afterwards the bag of $t_{u}^{\mathcal{T}^{\prime}}$ contains $u_{i+1}, u_{i+1}^{\square}, v_{1}$ and $v_{1}^{\square}$, thus covering the remaining edges. After this step we increased the width of $\mathcal{T}^{\prime}$ by at most 13. (Since the number of children of each tree decomposition node is at most two, this step enlarges every bag at most twice.) 
It is easy to verify that $\mathcal{T}^{\prime}$ is a valid tree decomposition of $G^{\prime}$. Furthermore, the width of $\mathcal{T}^{\prime}$ is at most the width of $\mathcal{T}$ plus 13.

We again get an analogous result for the exact variant.

- Corollary 16. Exact Defensive Alliance ${ }^{\mathrm{F}}$, parameterized by the treewidth of the graph, is $\mathrm{W}[1]$-hard.

\subsection{Hardness of Defensive Alliance}

We now introduce a transformation $\tau^{\mathrm{F}}$ that eliminates forbidden vertices. The basic idea is that we ensure that a forbidden vertex $f$ is never part of a solution by adding so many neighbors to $f$ that we could only defend $f$ by exceeding the bound on the solution size.

- Definition 17. We define a function $\tau^{\mathrm{F}}$, which assigns a Defensive Alliance instance to each Defensive Alliance ${ }^{\mathrm{F}}$ instance $I=\left(G, k, V_{\square}\right)$. For each $f \in V_{\square}$, we introduce new vertices $f^{\prime}, f_{1}, \ldots, f_{2 k}$. Now we define $\tau^{\mathrm{F}}(I)=\left(G^{\prime}, k\right)$, where $G^{\prime}$ is the graph defined by

$$
\begin{aligned}
& \mathrm{V}\left(G^{\prime}\right)=\mathrm{V}(G) \cup\left\{f^{\prime}, f_{1}, \ldots, f_{2 k} \mid f \in V_{\square}\right\}, \\
& \mathrm{E}\left(G^{\prime}\right)=\mathrm{E}(G) \cup\left\{\left(f, f_{i}\right),\left(f^{\prime}, f_{i}\right) \mid f \in V_{\square}, 1 \leqslant i \leqslant 2 k\right\} .
\end{aligned}
$$

We now prove that $\tau^{\mathrm{F}}$ yields a correct reduction from Defensive Alliance $^{\mathrm{F}}$ to Defensive Alliance.

- Lemma 18. Every Defensive Alliance ${ }^{\mathrm{F}}$ instance I has the same solutions as the Defensive Alliance instance $\tau^{F}(I)$.

Proof. Let $I=\left(G, k, V_{\square}\right)$ and $\tau^{\mathrm{F}}(I)=\left(G^{\prime}, k\right)$. Each solution $S$ of $I$ is also a solution of $\tau^{\mathrm{F}}(I)$ because the subgraph of $G$ induced by $N_{G}[S]$ is equal to the subgraph of $G^{\prime}$ induced by $N_{G^{\prime}}[S]$. Now let $S^{\prime}$ be a solution of $\tau^{\mathrm{F}}(I)$. For every $f \in V_{\square}$, neither $f$ nor $f^{\prime}$ are in $S^{\prime}$ because each of these vertices has at least $2 k$ neighbors, and $S^{\prime}$ cannot contain any $f_{i}$ because $N_{G^{\prime}}\left(f_{i}\right)=\left\{f, f^{\prime}\right\}$. Hence $S^{\prime}$ is also a solution of $I$ as the subgraphs induced by the respective neighborhoods are again equal.

We use $\tau^{\mathrm{F}}$ to show W[1]-hardness of Defensive Alliance by reducing from Defensive AlLIANCE $^{\mathrm{F}}$ while preserving bounded treewidth.

- Lemma 19. Defensive Alliance, parameterized by the treewidth of the graph, is W[1]hard.

Proof. Let $I=\left(G, k, V_{\square}\right)$ be a Defensive Alliance ${ }^{\mathrm{F}}$ instance, let $G^{\prime}$ denote the graph of $\tau^{\mathrm{F}}(I)$ and let $\mathcal{T}$ be an optimal nice tree decomposition of $G$. We build a tree decomposition $\mathcal{T}^{\prime}$ of $G^{\prime}$ by modifying a copy of $\mathcal{T}$ in the following way: For every $f \in V_{\square}$, we pick an arbitrary node $t$ in $\mathcal{T}$ whose bag $B$ contains $f$, and we add a chain of nodes $N_{1}, \ldots, N_{2 k}$ between $t$ and its parent such that, for $1 \leqslant i \leqslant 2 k$, the bag of $N_{i}$ is $B \cup\left\{f^{\prime}, f_{i}\right\}$. It is easy to verify that $\mathcal{T}^{\prime}$ is a valid tree decomposition of $G^{\prime}$. Furthermore, the width of $\mathcal{T}^{\prime}$ is at most the width of $\mathcal{T}$ plus two.

We again get an analogous result for the exact variant.

- Corollary 20. Exact Defensive Alliance, parameterized by the treewidth of the input graph, is $\mathrm{W}[1]$-hard. 


\section{Conclusion}

In this work, we proved that the problem of deciding whether a given graph possesses a nonempty defensive alliance whose size is at most a given integer is W[1]-hard when the parameter is the treewidth of the graph. This means that no fixed-parameter tractable algorithm exists under the common complexity-theoretic assumption $\mathrm{W}[1] \neq \mathrm{FPT}$. Still, recent work has shown the problem to be solvable in polynomial time on graphs of bounded clique-width [24], which implies that there is a polynomial-time algorithm for graphs of bounded treewidth. Our result proves that, for any such algorithm, the degree of this polynomial must necessarily depend on the treewidth unless W[1] = FPT. For future research it may be interesting to study related problems corresponding to other alliance notions such as offensive alliances.

\section{References}

1 Stefan Arnborg, Derek G. Corneil, and Andrzej Proskurowski. Complexity of finding embeddings in a k-tree. SIAM J. Algebraic Discrete Methods, 8(2):277-284, 1987.

2 Yuichi Asahiro, Eiji Miyano, and Hirotaka Ono. Graph classes and the complexity of the graph orientation minimizing the maximum weighted outdegree. Discrete Appl. Math., 159(7):498-508, 2011.

3 Bernhard Bliem and Stefan Woltran. Complexity of secure sets. CoRR, abs/1411.6549, 2014. Updated to version 3 on July 11, 2017.

4 Bernhard Bliem and Stefan Woltran. Complexity of secure sets. In Proc. WG, volume 9224 of LNCS, pages 64-77. Springer, 2015.

5 Hans L. Bodlaender. A tourist guide through treewidth. Acta Cybernet., 11(1-2):1-22, 1993.

6 Hans L. Bodlaender. A linear-time algorithm for finding tree-decompositions of small treewidth. SIAM J. Comput., 25(6):1305-1317, 1996.

7 Hans L. Bodlaender. Discovering treewidth. In Proc. SOFSEM, volume 3381 of LNCS, pages 1-16. Springer, 2005.

8 Hans L. Bodlaender and Arie M. C. A. Koster. Treewidth computations I. Upper bounds. Inform. and Comput., 208(3):259-275, 2010.

9 Aurel Cami, Hemant Balakrishnan, Narsingh Deo, and Ronald D. Dutton. On the complexity of finding optimal global alliances. J. Combin. Math. Combin. Comput., 58:23-31, 2006.

10 Chan-Wei Chang, Ma-Lian Chia, Cheng-Ju Hsu, David Kuo, Li-Ling Lai, and Fu-Hsing Wang. Global defensive alliances of trees and cartesian product of paths and cycles. Discrete Appl. Math., 160(4):479 - 487, 2012.

11 Marek Cygan, Fedor V. Fomin, Lukasz Kowalik, Daniel Lokshtanov, Dániel Marx, Marcin Pilipczuk, Michal Pilipczuk, and Saket Saurabh. Parameterized Algorithms. Springer, 2015.

12 Artan Dermaku, Tobias Ganzow, Georg Gottlob, Benjamin J. McMahan, Nysret Musliu, and Marko Samer. Heuristic methods for hypertree decomposition. In Proc. MICAI, volume 5317 of LNCS, pages 1-11. Springer, 2008.

13 Michael Dom, Daniel Lokshtanov, Saket Saurabh, and Yngve Villanger. Capacitated domination and covering: A parameterized perspective. In Proc. IWPEC, volume 5018 of LNCS, pages 78-90. Springer, 2008.

14 Rodney G. Downey and Michael R. Fellows. Parameterized Complexity. Monographs in Computer Science. Springer, 1999.

15 Rosa I. Enciso. Alliances in Graphs: Parameterized Algorithms and on Partitioning SeriesParallel Graphs. PhD thesis, University of Central Florida, Orlando, Florida, USA, 2009. 
16 Henning Fernau and Daniel Raible. Alliances in graphs: a complexity-theoretic study. In Proc. SOFSEM, volume 2, pages 61-70. Institute of Computer Science AS CR, Prague, 2007.

17 Henning Fernau and Juan A Rodríguez-Velázquez. A survey on alliances and related parameters in graphs. Electron. J. Graph Theory Appl. (EJGTA), 2(1):70-86, 2014.

18 Gary William Flake, Steve Lawrence, C. Lee Giles, and Frans Coetzee. Self-organization and identification of web communities. IEEE Computer, 35(3):66-71, 2002.

19 Jörg Flum and Martin Grohe. Parameterized Complexity Theory. Texts in Theoretical Computer Science. Springer, 2006.

20 Teresa W. Haynes, Stephen T. Hedetniemi, and Michael A. Henning. Global defensive alliances in graphs. Electr. J. Comb., 10, 2003.

21 Yiu Yu Ho and Ronald D. Dutton. Rooted secure sets of trees. AKCE Int. J. Graphs Comb., 6(3):373-392, 2009.

22 L. H. Jamieson. Algorithms and Complexity for Alliances and Weighted Alliances of Various Types. PhD thesis, School of Computing, Clemson University, Clemson, South Carolina, USA, 2007.

23 Lindsay H Jamieson, Stephen T Hedetniemi, and Alice A McRae. The algorithmic complexity of alliances in graphs. J. Combin. Math. Combin. Comput., 68:137-150, 2009.

24 Masashi Kiyomi and Yota Otachi. Alliances in graphs of bounded clique-width. Discrete Appl. Math., 223:91-97, 2017.

25 Ton Kloks. Treewidth: Computations and Approximations, volume 842 of LNCS. Springer, 1994.

26 András Kornai and Zsolt Tuza. Narrowness, pathwidth, and their application in natural language processing. Discrete Appl. Math., 36(1):87-92, 1992.

27 Petter Kristiansen, Sandra M Hedetniemi, and Stephen T Hedetniemi. Introduction to alliances in graphs. In Proc. ISCIS, pages 308-312. CRC Press, 2002.

28 Petter Kristiansen, Sandra M Hedetniemi, and Stephen T Hedetniemi. Alliances in graphs. J. Combin. Math. Combin. Comput., 48:157-178, 2004.

29 Rolf Niedermeier. Invitation to Fixed-Parameter Algorithms, volume 31 of Oxford Lecture Series in Mathematics and its Applications. Oxford University Press, Oxford, United Kingdom, 2006.

30 Neil Robertson and Paul D. Seymour. Graph minors. III. Planar tree-width. J. Combin. Theory Ser. B, 36(1):49-64, 1984.

31 Khurram H. Shafique and Ronald D. Dutton. Maximum alliance-free and minimum alliancecover sets. In Congr. Numer, volume 162, pages 139-146, 2003.

32 Stefan Szeider. Not so easy problems for tree decomposable graphs. CoRR, abs/1107.1177, 2011.

33 Mikkel Thorup. All structured programs have small tree-width and good register allocation. Inform. and Comput., 142(2):159-181, 1998.

34 Ismael González Yero and Juan A Rodríguez-Velázquez. Defensive alliances in graphs: a survey. CoRR, abs/1308.2096, 2013. 